\title{
Evaluation of Interfaces for IRS: Modelling End-User Searching Behaviour
}

\author{
Victoria Manglano, Micheline Beaulieu and Stephen Robertson \\ Centre for Interactive Systems Research, Department Information Science, City University \\ Northampton Square, London EC1V 0HB, UK \\ $\{\mathrm{vm}, \mathrm{mmb}, \mathrm{ser}\} @$ soi.city.ac.uk
}

\begin{abstract}
This paper describes an investigation in progress into the searching behaviour of end-users and how it is affected by interface design. The project focused on qualitative data gathering and heavily relies on thinkaloud protocols. 65 end-users were observed searching a bibliographic database (Medline) in their natural setting. The two most commonly used commercial versions of Medline were selected as experimental variable (OVID for Windows and WinSPIRS)- both use an underlying Boolean system and a Windowsbased interface, but present fundamental differences in terms of the use and the presentation of searching tools and results presentation. Marchionini's model of information seeking (1995) was used as a framework. The results showed that there are some discrepancies between this model and the behaviour observed. The results of the comparison of searching behaviour with the two systems have shown some fundamental differences, but also have provided some areas of similarities in behaviour which seem to indicate that there could be some genuine end-user characteristics which are independent of system and interface design.
\end{abstract}

\section{Research Objectives}

The overall objective of the current research is to investigate end-user searching behaviour and how it is affected by interface design. In other words, how the interface to an IRS affects the way users search for information and their performance, and how to evaluate systems to show this.

The research focuses on the end-users and follows what has been called "human approach" or "cognitive approach" in IR research [1,2], whose main focus is the interactive aspects of IR and the interaction between the user and the system, or better, between the user and the information. The aim of this human oriented IR research is to understand and describe the IR processes, to assist user training and searching, and contribute to the development of more effective IR interfaces and systems [3]. The need to include the user in the evaluation of IR systems has been recognised for a long time, and research focusing on the human elements within the IR processes has increased [4], as well as an increased interest in interactive aspects in some of the more traditional system oriented research (e.g. the inclusion of an "interactive track" within TREC experiments, [5]). Although there has been development of theoretical interactive IR models (e.g. [6]), there is still considerable research needed in this area. The relative infancy state of this type of research in IR, means that research methods (both collection and analysis methodologies) are still being developed, so considerable part of the current research has also involved the experimentation in data analysis techniques.

More specifically the objectives of the project are -

1) Investigate the searching behaviour of end-users in a bibliographic database; in particular, look

into how the searching session can be divided into subprocesses or stages. Marchionini's model of information seeking behaviour [7] has been used as a framework because it resulted from a series of studies carried out with different user populations and different information systems, and because it incorporates ideas put forward by previous studies in the user-centred tradition of IR research. Special attention has been paid to the transition between subprocesses and the iteration processes. Marchionini's model distinguishes the following stages in end-user searching: 1) Problem recognition and acceptance; 2) Problem definition; 3) Search system selection; 4) Query formulation; 5) Query execution; 6) Examination of results; 7) Extract information; 8) Reflection/Iteration/Termination. The data collected has provided a more detailed description of the specific stages and the different paths users take. We will discuss below some of the differences between the model we started with and the model resulting from the analysis of the data. 
Investigate to what extent the actual searching tool affects and modifies the searching processes, special attention was paid to presentation of searching tools or options and the presentation of information in the query formulation and reformulation stages, and the balance between system and user control over the searching session. Although the two systems selected for the observations of users are Boolean systems, they also have some fundamental differences in terms of the use and presentation of searching tools and results presentation. The results so far have shown some fundamental differences between the behaviour of the different samples, but, surprisingly have provided areas of similarities, which seem to indicate that there could be some genuine end-user characteristics which are independent of the system design.

\section{The Project: Selection of Application and Environment}

Medline, the electronic version of the bibliographic database produced by the National Library of Medicine (NLM) of the US, was selected as the basis of the research. Focusing on a single database reduces possible differences due to specific databases characteristics. Medline is one of the most frequently used databases within the medical field and in the broader area of electronic information as a whole. The fact that it is a subsidised database has facilitated the wide spread of different versions on different access media. Medline can be considered a rich bibliographic database as it contains long abstracts, a well-constructed hierarchical thesaurus (Medical Subject Headings or MESH) and different document types and other general categories limits.

The two most commonly used commercial versions of Medline were selected as experimental variableOVID for Windows from OVID Technologies and WinSPIRS from SilverPlatter Information. Both use an underlying Boolean system and a Windows-based interface $[8,9,10]$. The main differences between the two systems in terms of their interfaces were identified, and it was anticipated that these differences would produce significant users behavioural differences:

1. Subject search.

Both systems implement several ways of making use of MeSH thesaurus for searching, such as the availability of the thesaurus online, limiting the search to the MeSH field, and mapping facilities, but it was anticipated that some of them would not be used at all as in-depth training is needed. However, mapping facilities are provided in both systems, by which the system maps the natural language search query input by the user with the most relevant MeSH headings. The main difference between the two systems is that in OVID the mapping facility is automatically initiated by the system, and therefore subject search is a default; while in WinSPIRS the mapping facility needs to be user initiated, thus, textword search is the default searching option.

2. Intersect

One of the main characteristics of Boolean systems is the possibility of combining different query terms by way of using Boolean Operators. The presentation and syntax needed to use these is fundamentally different in the two systems studied- OVID provides a Combine command button and the Boolean operators AND and OR are presented in a pop up window for the user to select, the user only needs to double click on the search lines to be combined and select the relevant operator; on the other hand, WinSPIRS requires that the user types in the query terms (or query line number) and the operators. It was anticipated that given the more intuitive and less demanding implementation in OVID, the Intersect move would be more frequent in this application.

3. Presentation of results

There are significant differences in the way query results are presented in both applications: OVID provides independent windows for query formulation and results inspection, and the whole record is presented as a default, besides, the user needs to click on the View Set command button to display results. On the other hand, WinSPIRS integrates in the main screen the query formulation, search history and display of results areas. Display of results is automatic. A record citation is displayed as a default. It was anticipated that the integration of the searching and viewing of results tasks would be beneficial to the user. WinSPIRS provides a Lateral searching or hypertext type of search facility, by which the user can highlight any term from the display of results and add it to the search, it was anticipated that user would make use of this facility to carry out "Citation pearl growing" type of search strategies.

4. Lack of guidance on the search stages and reformulation aids

What both systems have in common is the limited guidance provided to the user in terms of what facilities are most appropriate at each stage, as all the searching facilities are presented at the same time, and limited query expansion or query reformulation aids. 
On the selection of the sites to carry out the empirical studies an attempt was made to get away from the purely academic environment, in order to try to get access to a variety of users -including doctors, academic and research staff, consultants, health professionals, nurses, media researchers, and members of the public.

Data was collected in 3 libraries in London:

1. St. George's Medical School Library (STG) (30 users)

This is a fairly big and very busy library. Although there is a focus on end-user searching, online searching and mediated searching is still being carried out. At the time of the study, a collection of 9 databases, including Medline (OVID for Windows software) were available over the Medical School Network, that could be accessed from the Library, Computer Unit or any computer linked to the network. 11 networked workstations were available in the library, with two dedicated workstations for database searching (a booking system applies to these two). Hospital staff and students normally receive formal training sessions, either in the workstations themselves or in a special purpose training room. Training was not compulsory, but was encouraged.

2. St. Mary's Medical School Library (STM) (20 users)

This is a medium size, busy library, that provides services to staff and students at St. Mary's Hospital and other Imperial College members (nurses and physiotherapists are excluded). Medline with SilverPlatter's WinSPIRS software was available via the Imperial College Network. Staff needed to come to the library to use it. Only one terminal was available in the library, for which a booking system was at work (this was placed in a private room in the library, that suited perfectly for the purpose of the observations). Training was provided on a one-to-one basis for those users that required it. A printed guide to searching was available next to the terminal.

3. The Health Care Information Service (SRIS, BL) (15 users)

As part of the Health Care Information Service, the Aldwych Reading Room houses the collection on the life sciences and technologies. It is a reference library, offering free end-user searching to a very exhaustive collection of 18 databases on CD-ROM, including SilverPlatter Medline (with both PCSPIRS and WinSPIRS software). It is a fairly busy public library with a variety of users including students (about half of the users), academic staff, users from companies, charities and the voluntary sector; media researchers; members of the public for personal interest and librarians or information specialists. The CD-ROM service includes 4 networked terminals providing a menu access to all databases. At the time of the observations, the library had recently updated one of the terminals to Windows-based software (WinSPIRS)- readers were invited use it, but the DOS-based software was available at the same time. They ran a booking system. Formal training was not provided as it is a public access library, but users were given a short introduction at the terminal.

\section{Methods of Data Collection and Analysis}

\subsection{Data Collection}

\begin{tabular}{|c|c|c|c|c|}
\hline User Status & STG (OVID) & $\begin{array}{l}\text { STM } \\
\text { (WinSPIRS) }\end{array}$ & $\begin{array}{l}\text { SRIS } \\
\text { (WinSPIRS) }\end{array}$ & TOTAL \\
\hline Hospital doctors & $37 \%$ (11 users) & $50 \%$ (10 users) & -- & $32 \%$ (21 users) \\
\hline Postgraduates & $23 \%$ (7 users) & $30 \%$ (6 users) & $13 \%$ (2 users) & $23 \%$ (15 users) \\
\hline Undergraduates & $13 \%$ (4 users) & $5 \%$ (1 user) & $13 \%$ (2 users) & $10 \%$ (7 users) \\
\hline Research Staff & $17 \%$ (5 users) & $15 \%$ (3 users) & $7 \%$ (1 user) & $14 \%$ (9 users) \\
\hline Nurses & $10 \%$ ( 3 users) & -- & -- & $5 \%$ (3 users) \\
\hline Consultants & -- & -- & $20 \%$ (3 users) & $5 \%$ (3 users) \\
\hline Writer/editor & -- & -- & $20 \%$ (3 users) & $5 \%$ (3 users) \\
\hline Industry & -- & -- & $20 \%$ (3 users) & $5 \%$ (3 users) \\
\hline Librarian & -- & -- & $7 \%$ (1 user) & $1 \%$ (1 user) \\
\hline Total & 30 users & 20 users & 15 users & $100 \%$ (65 users) \\
\hline
\end{tabular}




\section{Table 1: User Status of Study Participants}

The project focused on qualitative data gathering. Users in their natural setting were observed using the same database (Medline) with two different windows-based interfaces. A rich observation setting was selected in order to allow as many observations as possible. To avoid interference, users were invited to search for their own real information needs for as long or as short a time they needed to. The number of end-users observed was 65.

Although it could be argued that Medical School Libraries are still within the general environment of Academic users, the variety of types of users that participated in the study was very rich, undergraduate students represented only $10 \%$ of the sample. Besides the users from SRIS represent a different more broad type of users, including consultants and media and industry professionals.

The procedure of the user study was as follows:

Before searching, users were approached and invited to participate in the study, with detailed information on procedure, duration of the study and confidentiality. Once they had agreed, they were asked some questions (pre-search questionnaire) including details of status, experience with computers and computerised databases, training, purpose of search, and expectations about the content of the system. Users were asked to describe their information need.

While searching, users were asked to think aloud (and taped) describing what they were doing and what they understood about what the system was doing. A record of the actions and changes was also kept, for which a system of codes was devised. At the end of the search, the search history -generated automatically by the software- was saved to complement the data recorded.

Think-aloud protocols have been increasingly used in IR research as a means of complementing the external observations in studying the decision making processes involved in searching. This type of concurrent think aloud protocol is suitable to gather information on the user's intentions during the searching task, and it may be the only source of data on cognitive processes involved in using the system. These verbal protocols are very useful for determining the source of user's misconceptions and determining how sophisticated their conceptual models of the system are. Although the user cannot rationalise their explanations about what they are doing because it is a concurrent verbal report, they perhaps are more conscientious and take more time to carry out the tasks as a result of the think aloud and observation.

Despite the lack of any automatic means of recording the search session, such as logging facilities or video recording, the integration of the different type of data collected -the tape transcriptions, search sessions records and search histories- provided a very detailed and reliable record of the search session.

After searching, an interview next to the terminal was conducted (and taped) to assess the users' understanding of interface elements, their satisfaction with the interaction, and difficulties experienced while searching.

\subsection{Coding and Analysis}

Once the search sessions think-aloud protocols and interviews were transcribed in full, a coding system was devised for analysis purposes. The coding started with an initial list of codes that was theory-driven, based on FIDEL's moves [11] and BATES's search tactics [12], with the addition of system specific moves. Given the considerable methodological differences between those two studies and the current research, the final list of codes is very different than the ones they proposed- as far as we know, BATES work has not been validated empirically, and FIDEL's is based on the observation of professional searches. Moreover, the increasing complexity of systems has necessitated the addition of certain moves.

The list was iteratively refined, as well as the detailed definitions of each category, as the different search sessions were being coded, in order to account for differences in both systems. Moreover, a considerable amount of code types were data-driven as they evolved while the coding was carried out, this refers especially to what we have called "verbal reports", as well as codes in areas such as selection of terms from display or codes associated with relevance judgements. The model used as a framework was also inappropriate for certain stages for two reasons- some stages were not included such as Evaluation of number of results, and some stages were not detailed enough, such as Query Reformulation.

From the think-aloud protocols two types of codes were generated: Moves and Verbal Reports. Moves or Search Moves include any change in query formulation at any step, as well as other search stages. These were classified by the following stages:

- Choose Search System, in which the user selects one or more sets of data within the database; and also changes in the initial selection.

- Formulate Query (i.e. Initial Query), the users type in an initial search statement and associated selections for automatic mapping facility (OVID). 
- Query Reformulation, once the user has completed a cycle and viewed results; they can carry out moves of the following types, all these moves are user initiated in the two systems studied:

Refine- in which the user types in some kind of substitution terms/heading, such as a more general, more specific, related, opposite or synonym heading or textword.

Repeat- in which the user repeats the heading or term in some form (e.g. uses a subject heading as a textword or vice versa; repeats the same search term within the same data set or different data set; changes the selections associated to a subject heading).

Add- in which the users types in additional search terms, i.e. the terms represent an additional facet or subject as opposed to substitution terms of the Refine type.

Limit or Weight- to reduce the number of records retrieved the user applies different database specific limits such as documents in an particular language, limit by date, limit by type of document, etc.

Intersect, by which the user combines terms/headings representing another or additional query components.

- Examine Results, is a stage in which the users first evaluate the number of documents retrieved, before reviewing them; sometimes they start reviewing results although they express their inability to reduce the set size (Fail to Reduce), they view the results and carry out Relevance Assessments.

- Extract Information, stage in which users mark, write down, print or save results.

- Stop, users provide reasons why the stop searching whether or not they are satisfied with the results obtained.

On the other hand, Verbal Reports provide information as to what the user is thinking while searching. They have been classified by type (i.e. what information they provide) and by content (i.e. what search stage they refer to). This type of information is the result of the method of data collection used and provide an insight into the searching behaviour of end-users. This part of the analysis and the integration with other types of data collected is still in progress.

Search sessions were also divided into cycles, and number of terms used and source of terms were recorded.

\section{Preliminary Results}

\subsection{Introduction}

The analysis of the 65 end-user search sessions produced a search model with less stages than the one described in Marchionini's model [7]. On one hand, the two initial stages, i.e. Problem recognition and acceptance, and Problem definition, are not present in the search interactions observed. We could argue whether these stages occurred before the observation started, or whether they didn't exist. Although these stages are implicit in the user's descriptions of their subject need and, from questionnaire data, we know that most of the users know more about their information need than what they actually use during the search session, also it is clear that most of them do not carry out any preparation in advance. On the other hand, the distinction between stages 4 and 5, i.e. Query Formulation and Query Execution was not observed in the sample, from interview data we could argue that end-users do not perceive this distinction. These two main differences could be affected by interface design (i.e. the systems do not support more complex search processes) or be a genuine characteristic of end-users. These are areas in which interface and system design could be improved.

On the other hand, more detailed information in relation to certain stages and the transition between the different stages was collected. 
This is a comparative summary of the search stages (and progress) in the two models:

Marchionini's model (1995)

New model

1. Problem recognition and acceptance

2. Problem definition

3. Search system selection

4. Query formulation

5. Query execution
1. Selection of database or dataset (go to 2.1.)

2. Search

2.1. Initial Search (go to 3 or 4 or 5 )

2.2. Search Reformulation (go to 3 or 4 )

3. Evaluation of number of results

3.1. The system provides search results

(back to 2.2., or go to 4 or 5)

3.2. 0 hits results (back to 2.2.)

4. Intersect and Limit or Weight (go to 5 or back to 2.2.)

5. View (Go to 6)

6. Selection of documents to print or save

(back to 1 or 2.2. or go to 7)

8. Reflection/iteration/termination 7. Stop

\subsection{Some Initial Findings by Search Stage}

\subsubsection{Search}

\section{Very Low Incidence of Inconsistent Users (17 users - 26\% of Overall Sample)}

Users can be classified according to whether they used one searching approach only (named as consistent users) or whether they made use of subject search (i.e. thesaurus or mapping) and textword or free text search within the same searching session (inconsistent users). We could say that the users that follow just one approach can be defined as more consistent, while users that combine both approaches could be considered less consistent; the concept of consistency could also be applied to other behavioural decisions discussed below. Consistency in this context has no value judgement, i.e. being consistent is not necessarily a good thing.

\begin{tabular}{|l|l|l|}
\hline Sites & Consistent Users & Inconsistent Users \\
\hline $\begin{array}{l}\text { STG } \\
\text { (OVID) }\end{array}$ & $67 \%$ (20 users) & $33 \%$ (10 users) \\
\hline $\begin{array}{l}\text { STM } \\
\text { (WinSPIRS) }\end{array}$ & $80 \%$ (16 users) & $20 \%$ (4 users) \\
\hline $\begin{array}{l}\text { SRIS } \\
\text { (WinSPIRS) }\end{array}$ & $80 \%$ (12 users) & $20 \%$ (3 users) \\
\hline
\end{tabular}

\section{Table 2 : Search Consistency}

Overall results are very similar in the three samples. The issue here is the very low incidence of combination of approaches, in the two systems studied, that may be more appropriate in some instances. There is some evidence that consistent users may sometimes miss opportunities that may have yield better results. This is an area that needs attention and in which interface design can contribute. The slightly higher percentage of inconsistent users with the OVID system could be an indication that automatically providing a mapping facility to the controlled vocabulary could be an advantage, although previous experience with more traditional Boolean systems could also be an issue.

It is not obvious that inconsistent users have a plan or that they apply both textword and subject searches deliberately. For example, there are instances in which users combine in the same query textword and subject (e.g. STM). In fact, the data from the interviews and think-aloud indicates that these users are not always aware of the difference between subject vs. textword search and the implications in their results. There is a difference of degree with other users, at least they talk about MeSH and subject headings, although they may have slightly wrong assumptions about them (It is difficult to know whether users are more aware of the concept of subject search and the use of MeSH headings as a result of their exposure to a system like OVID that provides a system 
initiated automatic mapping facility, or it is just the result of their experience with Medline -and possibly related to the fact that these users started using Index Medicus in print, before the automation of Medline-). However, the 3 users from STG that described their searching strategy as starting with free text searches and using relevant $\mathrm{MeSH}$ headings in subsequent stages, belong to this group of inconsistent users.

\section{Users from St. George's are Consistent Using Subject Search vs. Users from both St. Mary's and SRIS that are Consistent Using Textword}

The most outstanding difference between the two systems is whether users are consistent in using textword or subject. The results below show that the default searching option, not surprisingly, is the one used more often. Therefore, from a systems point of view, the selection of default options becomes paramount. These results are highly significant, at the $0.1 \%$ level on a chi-square test. The small numbers involved raise a question mark over these results, however, it seems to be strong enough to overcome that objection.

\begin{tabular}{|l|l|l|}
\hline Sites & Subject Only & Textword Only \\
\hline STG (OVID) & $44 \%(13$ users $)$ & $23 \%(7$ users $)$ \\
\hline $\begin{array}{l}\text { STM } \\
\text { (WinSPIRS) }\end{array}$ & $5 \%(1$ user $)$ & $75 \%(15$ users $)$ \\
\hline $\begin{array}{l}\text { SRIS } \\
\text { (WinSPIRS) }\end{array}$ & -- & $80 \%$ (12 users) \\
\hline
\end{tabular}

Table 3 : Type of Search Consistency

There is a higher incidence of subject search in STG (OVID for Windows), which offers it as a default in the form of a system initiated mapping facility. The contrast here is between the $100 \%$ and $95 \%$ of the use of the default textword option in both WinSPIRS samples (STM and SRIS) vs. the 77\% percentage of use of the default option in OVID users (STG). This seems to indicate that previous experience and training in other systems, where textword is the norm, affects users behaviour. The issue of Negative Transfer Effect has not been studied in detail in IR, but it seems to be relevant, specially in the current environment of increasing use of IR systems and tools by end-users.

\section{Lower Subject Search Use (and Less Efficient) in WinSPIRS (STM and SRIS)}

The fundamental differences between the two systems studied in relation to the presentation of the subject search facility, both at the interface and search procedure levels, is the area in which end-user behaviour presents greater variations and contrasts.

\begin{tabular}{|l|l|l|}
\hline Sites & Subject Only & Subject + Textword \\
\hline STG (OVID) & $47 \%(14$ users $)$ & $77 \%(23$ users $)$ \\
\hline $\begin{array}{l}\text { STM } \\
\text { (WinSPIRS) }\end{array}$ & $5 \%(1$ user $)$ & $25 \%(5$ users $)$ \\
\hline $\begin{array}{l}\text { SRIS } \\
\text { (WinSPIRS) }\end{array}$ & -- & $20 \%(3$ users $)$ \\
\hline
\end{tabular}

\section{Table 4 : Subject Search}

Not only there is a substantially lower number of users in STM and SRIS that use subject facilities, but also they do it with less efficiency (for example, some of the users that opened the thesaurus failed to understand its structure and gave up). A test of statistical significance has not been performed on these results as the numbers are too small, however, these results are strongly supported by qualitative observation. It is difficult to ascertain whether the difficulties experienced by users are to do with the fact that the subject facilities are not automatic in WinSPIRS or with the differences in their specific implementations in both systems. 


\subsubsection{Query Reformulation}

\section{High Uptake of Reformulation Irrespective of Search System- 59 Users - 90\% of Sample}

In the three samples the uptake of reformulation of searches is very high irrespective of search system used, this could be considered an end-user characteristic as it is a user initiated move. This could indicate that neither of the systems satisfy users in their initial search, or that users like reformulating their search based on results of an initial search query. In the context of a Boolean system, the reformulation stage is very demanding for the end-user as the system does not take the initiative to reformulate or does not provide clues as to how reformulate.

\begin{tabular}{|l|l|}
\hline Sites & $\begin{array}{l}\text { Percentage and Number of } \\
\text { Users that Reformulate }\end{array}$ \\
\hline STG (OVID) & $93 \%(28$ users $)$ \\
\hline STM (WinSPIRS) & $95 \%(19$ users $)$ \\
\hline SRIS (WinSPIRS) & $80 \%(12$ users $)$ \\
\hline
\end{tabular}

Table 5 : Query Reformulation

\section{REPEAT Type of Reformulation Move is Higher than ADD (Except for SRIS)}

In relation to the type of reformulation moves carried out by users, there does not seem to be similarities between the two samples that used the same system. Both STG and STM seem to have higher percentage of REPEAT vs. SRIS which has higher percentage of ADD type of move. In this respect the variable perhaps could be experience with Medline. It is surprising that the sample with lower previous experience with Medline and searching in general, had the highest incidence of ADD type of move.

\begin{tabular}{|c|c|c|c|}
\hline $\begin{array}{l}\text { Reformulation } \\
\text { Moves }\end{array}$ & $\begin{array}{l}\text { STG } \\
\text { (OVID for Windows) }\end{array}$ & $\begin{array}{l}\text { STM } \\
\text { (WinSPIRS) }\end{array}$ & $\begin{array}{l}\text { SRIS } \\
\text { (WinSPIRS) }\end{array}$ \\
\hline REFINE & $47 \%$ (14 users) & $65 \%$ (13 users) & $40 \%$ (6 users) \\
\hline REPEAT & $73 \%$ (22 users) & $85 \%$ (17 users) & $53 \%$ (8 users) \\
\hline ADD & $60 \%$ (18 users) & $45 \%$ (9 users) & $\begin{array}{l}67 \%(10 \\
\text { users })\end{array}$ \\
\hline REPEAT+REFINE & $33 \%$ (10 users) & $20 \%$ (4 users) & $33 \%$ (5 users) \\
\hline REPEAT+ADD & $47 \%$ (14 users) & $10 \%$ (2 users) & $40 \%$ (6 users) \\
\hline ADD+REFINE & $30 \%$ (9 users) & -- & $33 \%$ (5 users) \\
\hline ONLY REPEAT & $13 \%$ (4 users) & $20 \%$ (4 users) & $7 \%$ (1 user) \\
\hline ONLY REFINE & $7 \%$ (2 users) & $10 \%$ (2 users) & -- \\
\hline $\begin{array}{l}\text { NO } \\
\text { REFORMULATION }\end{array}$ & $7 \%$ (2 users) & $5 \%$ (1 user) & $20 \%$ (3 users) \\
\hline ALL & $20 \%$ (6 users) & $35 \%$ (7 users) & $27 \%$ (4 users) \\
\hline
\end{tabular}

Table 6: Summary of Reformulation Moves per User

There seems to be a higher percentage of situations in which the users repeat the search term, in some form, rather than modifying it. This seems to indicate that end-users do not distinguish between formulating a query and executing a query (or searching), stages that most of the models of searching behaviour have traditionally distinguished, based on their observation of expert searches. However, given that the numbers are too small, a test of statistical significance has not been performed.

What the three sets have in common is the low percentage of users presenting the three types of reformulation moves : only 17 users, that represent a $26 \%$ of the overall sample. This could be an indication of how end-users perceive the search process as having just one path to follow, instead of all the possibilities available for reformulation. 


\subsubsection{Search Session Length}

\section{STM Users Carry out Longer Searches}

Search sessions were divided into search cycles in order to provide a means of classifying users according to the complexity of their searches and to compare users within and across samples. A search cycle is defined as from the point in which the user enters a query term to when he/she displays information retrieved (and prints or saves it). The number of cycles per search could be one measure of complexity of the search, although in some instances it could also be due to lack of experience, limitations of the system or indication of user's persistence.

\begin{tabular}{|l|l|l|l|}
\hline Number of Cycles & STG (OVID) & $\begin{array}{l}\text { STM } \\
\text { (WinSPIRS) }\end{array}$ & $\begin{array}{l}\text { SRIS } \\
\text { (WinSPIRS) }\end{array}$ \\
\hline 1 or 2 cycles & $50 \%$ (15 users) & $30 \%$ (6 users) & $47 \%$ (7 users) \\
\hline 3 or 4 cycles & $30 \%$ (9 users) & $45 \%$ (9 users) & $26 \%$ (4 users) \\
\hline 5 or more cycles & $20 \%$ (6 users) & $25 \%$ (5 users) & $27 \%$ (4 users) \\
\hline
\end{tabular}

Table 7: Summary of number of cycles per user

We seem to see some differences in behaviour, as grouped below, but the data is not statistically significant:

1) STG and SRIS results are similar (around 50\% of users carry out 1 or 2 cycles, the other half are evenly distributed in 3 or 4 cycles and 5 or more cycles). Given that these two groups used different systems the explanation of this results could have to do with the lack of experience in SRIS users, while for STG users, the success of the automatic mapping facility could explain the high percentage of users carrying out short searches.

2) STM users may need to carry out longer searches perhaps due to the lack of subject mapping facility; i.e. they struggle to get the results they need and add more terms.

\subsubsection{Number and Source of Terms per User}

\section{Higher Number of User Generated Terms in STM}

Details of the number and source of terms (i.e. user generated vs. system suggested) per user were recorded, in order to relate this with other system or user characteristics.

\begin{tabular}{|l|l|l|l|}
\hline Number of Terms & STG (OVID) & $\begin{array}{l}\text { STM } \\
\text { (WinSPIRS) }\end{array}$ & SRIS (WinSPIRS) \\
\hline 1 to 3 terms & $57 \%$ (17 users) & $25 \%(5$ users $)$ & $27 \%$ (4 users) \\
\hline 4 or 5 terms & $33 \%$ (10 users) & $25 \%$ (5 users) & $40 \%$ (6 users) \\
\hline more than 5 terms & $9 \%$ (3 users) & $50 \%$ (10 users) & $33 \%$ (5 users) \\
\hline $\begin{array}{l}\text { terms suggested by } \\
\text { system }\end{array}$ & $20 \%$ (6 users) & $50 \%$ (10 users) & $40 \%$ (6 users) \\
\hline
\end{tabular}

\section{Table 8: Summary of Number of Terms per User}

Overall WinSPIRS searchers use more terms in their searches, which represents a considerably increase in the cognitive load on the user to carry out the search. The results referring to number of terms used ( 3 first rows in table 8) are statistically significant at the 5\% level on a chi-square test. The interpretation of these numerical results is that this issue could be related with the lack of mapping facility and the fact that user generated terms are less successful in retrieving the necessary information. On the other hand, WinSPIRS users also make greater use of system suggested terms interactively (we mean here terms from the records) to try and define their information need. WinSPIRS users, as we have seen already, need to carry out longer searches and include more user generated terms than OVID users, which may be because there is not a system generated automatic mapping facility. 


\subsubsection{Evaluation of Number of Results or Magnitude Assessment}

Despite the fact that this stage has not been included in Marchionini's or other IR models previously proposed in the literature, think-aloud data provided repeated evidence that users carry out evaluation of number of hits repeatedly, before viewing results. Evaluation of number of results is a stage that motivates different actions according to the number of results provided by the system. The issue of size of the collection seems a relevant one in Medline, as compared to experimental collections. It seems that users spend more resources trying to get a manageable size of documents to inspect than thinking about the information itself. This is a common characteristic of all Boolean Systems that provide no way of ordering the retrieval output.

There is some indication that end-users handle size reducing moves more often and more efficiently than size enlarging moves. This has to be related to the classification of cycles as simple vs. complex, according to whether the user viewed results after typing in only one query term or whether they typed in more subsequent query elements before viewing results. The results in relation to type of queries are presented in the table below:

\begin{tabular}{|l|l|l|l|}
\hline $\begin{array}{l}\text { Number and Type of } \\
\text { Cycle }\end{array}$ & STG (OVID) & $\begin{array}{l}\text { STM } \\
\text { (WinSPIRS) }\end{array}$ & SRIS (WinSPIRS) \\
\hline total no. cycles & 97 cycles & 80 cycles & 50 cycles \\
\hline mean per user & 3.2 cycles per user & 4 cycles per user & 3.3 cycles per user \\
\hline$\%$ (no.) simple cycles & $26 \%(25)$ & $16 \%(13)$ & $36 \%(18)$ \\
\hline$\%$ (no.) complex cycles & $74 \%(72)$ & $84 \%(67)$ & $64 \%(32)$ \\
\hline
\end{tabular}

\section{Table 9: Number and Type of Cycle per User}

These results seem to indicate that system's differences are not the only criteria that need to be taken into account. As with the number of cycles per user, STG and SRIS users present more similarities, while STM presents a higher percentage of complex cycles and mean per user.

\section{Higher Number of 0 Hits in WinSPIRS Samples (STM and SRIS)}

Instances of 0 hits results are considerably more frequent in both WinSPIRS samples. The higher use of textword search has to be related to this high incidence of 0 hits results.

\begin{tabular}{|l|l|}
\hline Sites & $\begin{array}{l}\text { Number of users (and } \\
\text { percentage) that get 0 hits }\end{array}$ \\
\hline STG (OVID) & $\begin{array}{l}20 \% \\
6 \text { users in 21 instances }\end{array}$ \\
\hline STM (WinSPIRS) & $\begin{array}{l}70 \% \\
14 \text { users in 52 instances }\end{array}$ \\
\hline SRIS (WinSPIRS) & $\begin{array}{l}73 \% \\
11 \text { users in 27 instances }\end{array}$ \\
\hline
\end{tabular}

Table 10: Number of Users that get 0 hits

\subsubsection{Intersect and Limit or Weight}

\section{Similar Use of Intersect Despite Systems Differences}

According to the high percentage of use of Intersect move in both STG and STM samples, we could say that Intersect is a Boolean system facility that end-users seem to master. Interview data confirms that a high percentage of users described their information need as a combination of concepts (already known to be linked somehow in the real world, or the search session being an attempt to assess whether that combination had been described before). On the other hand, the lower incidence of intersect in SRIS could be related to lower users experience. 


\begin{tabular}{|l|l|}
\hline Sites & $\begin{array}{l}\text { Number of users (and } \\
\text { percentage) that Intersect }\end{array}$ \\
\hline STG (OVID) & $\begin{array}{l}60 \% \text { (18 users) + } \\
3 \text { users intention }\end{array}$ \\
\hline STM (WinSPIRS) & $\begin{array}{l}75 \%(15 \text { users) + } \\
3 \text { users intention }\end{array}$ \\
\hline SRIS (WinSPIRS) & $\begin{array}{l}40 \%(6 \text { users) + } \\
3 \text { users intention }\end{array}$ \\
\hline
\end{tabular}

\section{Table 11: Intersect Results}

The substantial difference between the two systems studied in the presentation of the option and the syntax needed to use it seems to be irrelevant, as in both systems the experienced users learn to use the option whatever its presentation. Besides, in both systems we find similar number of users that intend to combine terms and fail for lack of system knowledge.

It is interesting to see that even though the mechanics of Intersect is more complex in WinSPIRS as users need to learn the syntax and use the Boolean operators, it is used as often, and experienced user learn to use it and feel comfortable with it.

\section{Lower Incidence of Limit or Weight as Compared to Intersect}

Limit and Weight moves allow the user to specify certain attributes of the documents to be retrieved that do not refer strictly speaking to their subject content, but refer to attributes such as language (e.g. English only), date, document type, and a set of Medline specific general categories known as checktags, such as age groups; male vs. female; human vs. animal, etc.

\begin{tabular}{|l|l|l|l|}
\hline Moves & $\begin{array}{l}\text { STG } \\
\text { (OVID) }\end{array}$ & $\begin{array}{l}\text { STM } \\
\text { (WinSPIRS) }\end{array}$ & $\begin{array}{l}\text { SRIS } \\
\text { (WinSPIRS) }\end{array}$ \\
\hline LIMIT/WEIGHT & $37 \%$ (11 users) & $20 \%$ (4 users) & $27 \%$ (4 users) \\
\hline INTERSECT & $60 \%$ (18 users) & $75 \%$ (15 users) & $40 \%$ (6 users) \\
\hline BOTH & $20 \%$ (6 users) & $20 \%$ (4 users) & -- \\
\hline NONE & $20 \%$ (6 users) & $25 \%$ (5 users) & $33 \%$ (5 users) \\
\hline
\end{tabular}

\section{Table 12: Intersect and Limit or Weight Moves Results}

As compared with Intersect results, a lower percentage of users apply Limits or Weights as part of their searches, although presentation of both options is similar in OVID, as both Intersect and Limit are provided as command buttons; and more obvious in WinSPIRS where only Limit is provided as a command button.

There is a very low percentage of users that include both types of moves in their search session - 10 user (15\% of the total of the sample). (20\% for STG and STM and none for SRIS). As with other system's options, it might be that users perceive that they just have one path to follow. On the other hand, there is a considerable number of users that do not use any of these type of set size reducing moves (16 users- $25 \%$ of the total sample). This could be due to lack of appropriate system presentation of the option or initiative in the query building process.

\subsubsection{Viewing Results}

\section{Similar Viewing Behaviour in the Three Samples Despite the Differences in Results Presentation and Defaults}

Viewing Results is a central stage in the search process, in which the user spends considerable amount of time (from a few minutes to up to 20 or 40 minutes). Users make individual decisions about each document as to whether they are relevant or not and monitor the search progress according to the results obtained. Most of the users were very selective. 


\section{Relevance Assessment is Based on Several Record Elements and it is Influenced by Several Factors}

Titles are the first source for relevance assessment, all users read the titles aloud or explained they were reading the titles. Abstracts are often read to help decide on the relevancy of a document, when the title is not very informative or clear, when the user does not know what something means, or when the paper is in a foreign language. Others sources of information to make relevance judgements were: age group, author, animal vs. human studies, country of publication or country as subject, date, journal, language, document type, institution, local holdings, ISSN.

Think aloud protocols provided information on other factors or criteria that could affect relevance judgements, such as Level or approach of treatment of the subject; papers already known by the user; Papers relevant for other reasons; papers already seen in the search session; sometimes users explained that they were missing documents they knew existed but were not coming up in the retrieved set.

\section{Very Low Use of MeSH Headings Interactively}

Despite the different presentation of MeSH headings in both systems, their interactive use as part of the viewing of results is very low in all the sample $(17 \%, 11$ users). This area is the most outstanding example of missed opportunities. This is in clear contrast with the stage in which users are presented with MeSH headings automatically as part of the mapping facility, in which qualitative observation shows that users select terms for searching effectively. In order to support the use of MeSH headings as a searching aid for reformulation, changes could be suggested both in terms of system and interface facilities, such as presenting MeSH headings independently of the rest of the records, and inviting users to select some of them for further searching, in some form of relevance feedback facility.

\begin{tabular}{|l|l|}
\hline Sites & $\begin{array}{l}\text { Number of users and } \\
\text { percentage that use MeSH } \\
\text { Headings Interactively }\end{array}$ \\
\hline STG (OVID) & $10 \%$ (3 users) \\
\hline STM (WinSPIRS) & $25 \%$ (5 users) \\
\hline SRIS (WinSPIRS) & $20 \%$ (3 users) \\
\hline
\end{tabular}

Table 13: MeSH Headings Use

\subsubsection{Stop}

Users normally stop after having selected at least a few references to documents they could read, having tried different approaches in searching and different terms and combinations. Users expressed their satisfaction with the documents extracted and explained they would read them, follow up references included in those papers, think about the information and perhaps may come back to search in the future. However, there are small numbers of users (10 users- 15\% of the total sample) that gave up searching or did not print out references for several reasons (e.g. because they had too many references to view or because they could not find the information wanted in STG; while in STM and SRIS they were having problems with the system syntax).

There is some indication that users perceive their search session as an ongoing process, which means that once they have some references to inspect they are happy to stop searching and read them and maybe come back in the future (time constraints and availability of systems could also affect this behaviour). This behaviour is consistent with human information processing abilities, users indicated that they have enough information to inspect for the time being. 


\section{Discussion and Conclusions}

In this study in progress, we investigated the searching behaviour of end-users in their natural setting and how it is affected by interface design. From the model point of view, the data collected has provided a more detailed description of the specific stages in the searching session and the different paths users take, as compared with the model used as a framework. From the observations, the two initial stages Problem Recognition and Acceptance; and Problem Definition are not apparent, and I there is some evidence that they do not occur as a separate stage. Besides, there is no evidence that end-users perceive a distinction between Query Formulation and Query Execution as defined in Marchionini's model.

The comparison of two different interfaces to the same database has provided evidence of two types of phenomena: 1) There is a series of searching behaviours that are common to users of both systems, such as the high percentage of combinations of terms (Intersect Move), the high uptake of the reformulation moves, and the similarities in viewing behaviour, including the stage in which end-user evaluate the number of hits obtained, these behaviours could be regarded as end-user characteristics; 2) There are areas of behaviour in which the different samples differ significantly, such as the difference in the use of subject searches, the differences in search session length, and number and type of terms used. These areas are all related to the main differences between the two systems as described in section 2 .

These findings need to be complemented and integrated with the analysis of the Verbal Reports and the Interview results. All these qualitative data will provide a richer framework for interpretation and discussion of the results.

\section{Reference List}

1. Ingwersen P. Information Retrieval Interaction. Taylor Graham, London, 1992.

2. Spink A. Information Science: A Third Feedback Framework. Journal of the American Society for Information Science 1997; 48 (8): 728-740.

3. Spink A. op. cit.

4. Beaulieu M. Experiments on interfaces to support query expansion. Journal of Documentation 1997; 53 (1): 8-19.

5. Beaulieu M, Robertson S, Ramussen E. Evaluating Interactive Systems in TREC. Journal of the American Society for Information Science 1996; 47 (1): 85-94.

6. Ingwersen P. Cognitive perspectives of information retrieval interaction. Journal of Documentation 1996; $52(1): 3-50$.

7. Marchionini G. Information Seeking in Electronic Environments (Cambridge Series on Human-Computer Interaction: 9). Cambridge University Press, Cambridge, 1995.

8. Jacsó, P. OVID/Medline: a gourmet choice, Database 1994; 17 (1): 31-38.

9. Jacso, P. WinSPIRS: windows software for SilverPlatter CD-ROMS, Online 1995; 19 (1): 74-81.

10. Manglano Bosch, V. and Hancock-Beaulieu, M. CD-ROM user interface evaluation: the appropriateness of GUIs, Online and CD-ROM Review 1995; 19 (5): 255-270.

11. Fidel R. Moves in online searching. Online Review 1985; 9 (1): 61-74.

12. Bates MJ Information Search Tactics. Journal of the American Society for Information Science 1979; 30 (4): 205-214. 